\title{
Motivos de uso de las redes sociales virtuales: Análisis de perfiles de mujeres rurales
}

\author{
Use motives of social networking sites: Analysis of rural womens' profiles
Jiménez-Cortés, Rocío; Rebollo-Catalán, $\quad \mathbf{M}^{\mathrm{a}} \quad$ Ángeles; García-Pérez, Rafael; Buzón-García, Olga
Universidad de Sevilla

\begin{abstract}
Resumen
En el presente trabajo, se muestran los resultados de una investigación cuyo objetivo es conocer los motivos de uso de las redes sociales virtuales de las mujeres rurales y determinar perfiles en función de sus respuestas. Para ello, se realiza un estudio de encuesta, cuyo cuestionario se aplica a 478 mujeres de entornos rurales de Andalucía de 18 a 65 años. Los resultados muestran dos perfiles de mujeres en función de sus motivaciones de uso de las redes, encontrando que las que usan las redes con una mayor variedad de motivos, muestran mayores niveles en intensidad, habilidad y autonomía de uso, mientras que las que la usan exclusivamente por motivos relacionales presentan niveles inferiores en estas variables. Esto sugiere que los motivos de uso es una variable explicativa de la inclusión digital de las mujeres rurales en las redes sociales. Los resultados permiten repensar las propuestas educativas de alfabetización digital
\end{abstract}

Fecha de recepción 21 de Junio de 2014

Fecha de aprobación 1 de Marzo de 2015

Fecha de publicación 22 de Marzo de 2015

\section{Palabras clave:}

Redes sociales, mujer rural, inclusión digital, estudios de las mujeres, capital social, motivos de uso, alfabetización digital, aprendizaje permanente

\begin{abstract}
This paper reports some of the findings from a study that aimed to analyse the motives for using social networking sites and to establish profiles from their responses. To do this, we carried out a survey study based on a purposely designed questionnaire answered by 478 women from rural areas of Andalusia aged 18 to 65. From an analysis of the responses, we identified two profiles based on the use motives of social networking sites. The results indicate that women, who use social networks with a higher diversity of uses, also express higher level of intensity, ability and autonomy of use. In contrast, women who use only social networks with a relational purpose, also express lower level of intensity, ability and autonomy in the use of social networks. These findings suggest that use motives is a predictor variable of rural women's digital inclusion in the social networks. The findings allow us to rethink the educational proposals of digital literacy.
\end{abstract}

Reception Date 2014 June 21

\section{Approval Date} 2015 March 01

Publication Date: 2015 March 22

\section{Keywords:}

Social networking sites, rural women, digital inclusion, women's studies, social capital, motives of use, digital literacy, lifelong learning

Esta investigación se centra en estudiar las motivaciones de uso de las redes sociales virtuales en mujeres de zonas rurales y descubrir perfiles de uso, con la finalidad última de repensar las propuestas educativas orientadas a la alfabetización digital de las mujeres para el ejercicio de una ciudadanía digital activa. Estudios internacionales indican que las redes sociales virtuales han cambiado la presencia y participación de las mujeres en los entornos virtuales (Clipson, Wilson \& 
DuFrene, 2010; Mazman \& Usluel, 2011). Las redes sociales son consideradas un contexto idóneo para el aprendizaje (Dabbagh \& Kitsantas, 2012), resultando clave indagar en los procesos por los cuales las personas aprenden en estos nuevos entornos y cuáles son sus motivaciones para hacerlo. Esto abre posibilidades para aprovechar las redes sociales en la formación y tender puentes entre lo formal y lo informal (Greenhow \& Robelia, 2009).

La mayor parte de las investigaciones realizadas sobre alfabetización digital y nuevas alfabetizaciones para el ejercicio de una ciudadanía digital activa y participativa (Area \& Pessoa, 2012; Coiro, Knobel, Lankshear \& Leu, 2008; Livingstone, Couvering \& Thuminn, 2008; Meyers, Erikson \& Small, 2013) insisten en la importancia de considerar los motivos de uso como una variable clave en el aprendizaje y la formación mediadas por tecnologías digitales interactivas. De ahí, la existencia de todo un conjunto de trabajos (Arteaga, Cortijo \& Javed, 2014; García, López de Ayala \& Catalina, 2013; Gómez, Roses \& Farias, 2012; Greenhow \& Burton, 2011) que indagan en los motivos de uso en relación con el aumento de capital social y su relevancia para el diseño de propuestas educativas, especialmente en lo que se refiere a las redes sociales como redes de aprendizaje (Sloep \& Berlanga, 2011) o como parte de los entornos personales de aprendizaje (Attwell, 2007).

Por otro lado, un amplio número de investigaciones sobre hábitos y experiencias de uso de las TIC se han centrado en poblaciones infantiles y juveniles (Chen, Hsiao, Chern \& Chen, 2014; Livingstone \& Helsper, 2007; Staksrud, Ólafsson \& Livingstone, 2013), pocas en personas adultas (Godfrey \& Johnson, 2009; Martinez-Pecino, Delerue \& Silva, 2013; Pfeil, Arjan \& Zaphiris, 2009), menos en mujeres (Castaño, 2009; Rubio y Escofet, 2013) y menos aún en mujeres de entornos rurales (Falk \& Kilpatrick, 2000; Rebollo \& Vico, 2014). Algunos informes advierten de las especiales dificultades que afrontan las comunidades rurales (LaRose, Gregg, Strover, Straubhaar \& Carpenter, 2007; Novo-Corti, VarelaCandamio \& García-Alvarez, 2014) y las personas mayores (Braun, 2013; Dias, 2012) en el acceso $y$ uso de las tecnologías, señalando los múltiples factores que pueden incidir en su exclusión digital. De ahí la relevancia que tiene en la actualidad la formación digital de la población, especialmente, aquella con mayor riesgo de exclusión digital, como son las mujeres del medio rural y las mujeres mayores.

En este sentido, este estudio persigue: a) conocer los motivos de uso que hacen las mujeres de zonas rurales de las redes sociales virtuales, b) determinar y caracterizar perfiles de mujeres en el uso de las redes sociales. Nuestra hipótesis principal es que los motivos de uso de las redes sociales es una variable significativa en la inclusión digital de las mujeres rurales, siendo las que las usan con una variedad de motivos de uso, las que presentan mayor grado de intensidad, habilidad y autonomía de uso.

\section{Antecedentes}

El uso de Internet como factor de inclusión digital es explorado desde distintas vertientes en la literatura científica. Así se ha abordado desde la perspectiva de las motivaciones de uso (Lin \& Lu, 2011; Seidman, 2013; Tosun, 2012; Vergés, 2012; Yang \& Brown, 2013), los tipos de uso (Carpenter, Green \& LaFlan, 2011; Cheung, Chiu \& Lee, 2010, Zillien \& Hargittai, 2009), la intensidad de uso (Hargittati \& Hsieh, 2010; Steinfield, Ellison \& Lampe, 2008) y las habilidades de uso (Hargittai, 2010; Hsieh, 2012).

Diversos estudios han analizado las motivaciones de uso de las TIC, en general (Vergés, Hache \& Cruells, 2011; Vergés, 2012) y de las redes sociales, en particular (Hew \& Cheung, 2012; Subrahmanyam, Reich, Waechter \& Espinoza, 2008; Lin \& Lu, 2011; Steinfield, Ellison \& Lampe, 2008). Entre estos últimos, también encontramos investigaciones específicamente centradas en las motivaciones de uso de Facebook (Arteaga, Cortijo \& Javed, 2014; Dogruer, Menevis \& 
Eyyam, 2011; Joinson, 2008; Nadkarni \& Hofmann, 2011; Seidman, 2013; Smock, Ellison, Lampe \& Wohn, 2011; Tosun, 2012; Yang \& Brown, 2013). Los motivos de uso son una variable clave en esta investigación ya que se abordan en el estudio del aprendizaje y la formación mediadas por tecnologías digitales interactivas (Coiro et al., 2008; Livingstone, Couvering \& Thuminn, 2008; Area \& Pessoa, 2012).

Todas estas investigaciones han mostrado una variedad de motivaciones con las que las personas usan las redes sociales. Basándose en Papacharissi \& Mendelson (2011), Smock et al. (2011) identificaron nueve motivaciones de uso de Facebook: como forma de pasatiempo habitual, para entretenerse de forma relajante, para compartir información expresiva, para evadirse, por compañerismo, para la promoción profesional, para la interacción social y para conocer gente nueva. Otras investigaciones también han encontrado motivaciones de uso muy similares (Arteaga et al., 2014; Dogruer et al., 2011; Hew \& Cheung, 2012; Yang \& Brown, 2013), identificando al menos tres tipos de motivaciones: orientadas a las relaciones sociales, al intercambio de información y al entretenimiento. Por su parte, Joinson (2008) también añade como principal motivación el refuerzo de la propia identidad.

En definitiva, las investigaciones (Lin \& Lu, 2011; Rebollo \& Vico, 2014) han revelado que las principales motivaciones en el uso de las redes sociales son relacionarse socialmente, es decir, mantener contactos con amigos y familiares y entretenerse, disfrutar y relajarse. Esto implica que las redes abren oportunidades para obtener recursos y apoyo de los contactos de la red, contribuyendo por tanto al aumento del capital social (Ellison, Steinfield \& Lampe, 2007; Valenzuela, Park \& Kee, 2009). Así el estudio de Ellison, Steinfield y Lampe (2007) centrado en estudiantes de pregrado analizan como cuando las circunstancias vitales cambian, como es el paso de secundaria a la universidad, y con ellas, cambian también las comunidades, los contactos y las relaciones, el capital social inicial se mantiene gracias a las redes sociales. Este capital es clave para el aprendizaje al contar con personas en las que confían para resolver sus problemas o incluso para conocer experiencias de otros compañeros que sirven de referentes en la nueva andadura. Así mismo, Sloep y Berlanga (2011) consideran relevante el papel que juegan las redes sociales como redes de aprendizaje especialmente por el capital social que deriva de la confianza entre los participantes y del apoyo entre compañeros.

En su estudio sobre perfiles de uso, Pfeil et al. (2009) encuentran una brecha de capital social en función de la edad, encontrando que mientras los adolescentes tienen redes más amplias de amigos (la mayoría de su misma edad), la red social de las personas mayores es más reducida aunque tiende a incluir personas de edades más diversas; también encuentran que los adolescentes hacen más uso de diferentes medios y más autorreferencias cuando se describen a sí mismos que las personas mayores. La brecha generacional en el uso de tecnologías digitales ha sido ampliamente mostrada en investigaciones previas (DiMaggio et al., 2004; Freese, Rivas \& Hargittai, 2006) siendo más probable encontrar personas no usuarias o usuarias funcionales entre las de mayor edad (Brandtzæga, Heim \& Karahasanovic, 2011).

Las investigaciones sobre inclusión digital también han documentado la relación entre motivación y habilidad y autonomía en el uso de las TIC (Hargittai; 2010; Hargittai \&y Walejko, 2008; Livingstone \& Helsper, 2010), habiéndose centrado la mayor parte de ellas en las habilidades digitales autopercibidas (van Dijk, 2006). En su estudio con adolescentes, Hargittai (2010) demuestra que la habilidad digital está positivamente asociada con los tipos de uso, encontrado que las personas que usan Internet de forma más diversa son aquellas que se perciben con un elevado nivel de habilidades, mientras que quienes se consideran menos hábiles son también quienes usan Internet de formas menos diversas. Los resultados de Livingstone y Helsper (2010) sugieren que las diferencias en las habilidades digitales se asocian a usos diferenciados de la 
Web de manera que aquellas personas que perciben que tienen un elevado nivel de habilidades usan de forma también más diversa Internet. Hargittai y Hinnant (2008) muestran que las personas con mayor nivel educativo y con antecedentes ricos en recursos usan la Web para más tipos de actividad, revelándose la habilidad un factor mediador en los tipos de actividades que la gente realiza en Internet.

Paralelamente, la investigación ha demostrado que el uso diversificado de las redes se asocia con una mayor autonomía e intensidad de uso (Hargittai \& Hinnant, 2008. Algunos estudios (Hargittai \& Walejko, 2008; Correa, 2010) encuentran que la relación del género con las actividades de creación y difusión de contenidos online están mediadas por la habilidad, aportando resultados que sugieren que incluso cuando el acceso y usos funcionales de las tecnologías se ha alcanzado, existe una brecha de participación entre quienes realizan actividad de creación y difusión de contenidos en Internet. Estos trabajos también apuntan a la importancia del estatus socioeconómico y, en especial, al nivel de estudios de los padres, como importante predictor de cómo la gente incorpora la Web en su vida cotidiana. Mayores niveles de educación parental están asociados a niveles más elevados de habilidad de uso de la Web, si se es hombre y blanco. Livingstone y Helsper (2007) encuentran también que la autonomía de uso está asociada con el tiempo de conexión a Internet.

La intensidad de uso también parece mostrarse una variable asociada a formas diversificadas de uso de las redes (Ellison et al., 2007; Stenfield et al., 2008; Valenzuela, Park \& Kee, 2009). En su estudio sobre la participación de estudiantes universitarios en las redes sociales, Hargittai (2008) pone de manifiesto que los contextos de uso y la experiencia con el medio están asociados a usos diversos, encontrando que los estudiantes que acceden a Internet en casa de un familiar o amigo tienen más probabilidades de un uso más autónomo de las redes. Correa (2010) plantea que una mayor experiencia en la red y formas más diversificadas de uso conllevan el desarrollo de habilidades más avanzadas. Hargittai (2010) también muestra que pasar más tiempo en línea lleva a un uso más diversificado de las redes. Los trabajos que han analizado la intensidad de uso de las redes sociales en relación con formas variadas de uso y, especialmente, con usos avanzados de las mismas también han documentado diferencias significativas entre hombres $\mathrm{y}$ mujeres (Hargittati \& Hsieh, 2010; Imhof, Vollmeyer \& Beierlein, 2007; Jackson, Ervin, Gardner \& Schmitt, 2001).

\section{Objetivos}

A la vista de la importancia que los motivos de uso tienen para la inclusión y el aprendizaje en red, nos planteamos averiguar las motivaciones que las mujeres de entorno rural expresan en el uso de las redes sociales con el fin de identificar perfiles en base a las mismas. Como hemos señalado con anterioridad, la mayor parte de las investigaciones sobre motivos de uso de las redes sociales se han realizado en niños y jóvenes y escasas se han realizado en adultos $y$ aún menos de comunidades rurales, siendo un colectivo diana en las políticas de inclusión digital de la región. El hecho de que la brecha digital de género en cuanto al acceso se haya reducido en la web 2.0 y especialmente en lo que se refiere al uso de las redes sociales virtuales motiva este estudio. La hipótesis de partida que inspira nuestro estudio es que los motivos de uso es una variable explicativa de la inclusión digital, considerando como indicadores de inclusión: la intensidad, habilidad y autonomía de uso en las redes sociales.

Por todo ello, los objetivos específicos que nos planteamos con nuestro trabajo son:

1. Describir los motivos de uso de las redes sociales virtuales por parte de las mujeres andaluzas de zonas rurales.

2. Determinar perfiles de mujeres en función de su motivación de uso de las redes.

3. Caracterizar dichos perfiles en función de la habilidad, autonomía e intensidad de uso. 


\section{Método}

\section{Participantes}

Participan en el estudio 478 mujeres andaluzas de zonas rurales de 18 a 65 años seleccionadas mediante un muestreo no probabilístico por cuotas en base a la variable edad, utilizando un procedimiento de afijación proporcional y, contemplando el nivel educativo como variable complementaria. Estas dos variables se han revelado muy explicativas junto con la localización geográfica de la brecha digital en estudios previos y, de ahí, considerarlas de forma complementaria en la estrategia de selección de nuestra muestra mujeres adultas de zonas rurales.

Se consideran los siguientes criterios de inclusión: a) tener su residencia y actividad habitual vinculada a una zona rural para lo que consideramos como espacios rurales los municipios con población inferior a 30.000 habitantes y densidad inferior a 100 habitantes por kilómetro cuadrado (ley 45/2007 de 13 de diciembre para el desarrollo sostenible del medio rural), b) tener una experiencia mínima de uso de una red social virtual de un año y, c) representar distintos perfiles de mujeres por edad, nivel educativo, situación familiar, etc.

La siguiente Tabla 1 resume las principales características de la muestra.

TABLA 1: Datos de las mujeres de la muestra del estudio

\begin{tabular}{|c|c|c|c|}
\hline Variables & Categorías & Frecuencias & Porcentaje \\
\hline \multirow[t]{4}{*}{ Edad } & $\leq 25$ años & 117 & $24.7 \%$ \\
\hline & 26 a 39 años & 153 & $32.3 \%$ \\
\hline & 40 a 54 años & 152 & $32.1 \%$ \\
\hline & > 55 años & 51 & \\
\hline \multirow[t]{4}{*}{ Nivel Educativo } & Sin estudios & 60 & $12.7 \%$ \\
\hline & Primaria & 170 & $34,8 \%$ \\
\hline & Secundaria (PO) & 56 & $11.8 \%$ \\
\hline & Universidad & 188 & \\
\hline \multirow[t]{5}{*}{ Situación laboral } & Empleo remunerado & 224 & $48.8 \%$ \\
\hline & Desempleada & 100 & $21.8 \%$ \\
\hline & Estudiante & 58 & $12.6 \%$ \\
\hline & Ama de casa & 54 & $11.8 \%$ \\
\hline & Jubilada/pensionista & 23 & \\
\hline \multirow[t]{4}{*}{ Estado civil } & Soltera & 204 & $42.7 \%$ \\
\hline & Casada & 229 & $47.9 \%$ \\
\hline & Divorciada/Separada & 32 & $\begin{array}{l}6.7 \% \\
27 \%\end{array}$ \\
\hline & Viuda & 13 & $2.7 \%$ \\
\hline \multirow[t]{2}{*}{ Hijos } & $\mathrm{Si}$ & 205 & $44.1 \%$ \\
\hline & No & 260 & $55.9 \%$ \\
\hline \multirow[t]{2}{*}{ Nacionalidad } & España & 463 & $96.9 \%$ \\
\hline & Otras & 10 & $2.1 \%$ \\
\hline \multirow[t]{4}{*}{ Redes sociales que usan } & Facebook y Tuenti & 206 & $43.7 \%$ \\
\hline & Solo Facebook & 196 & $41.6 \%$ \\
\hline & Solo tuenti & 45 & $\begin{array}{l}9.6 \% \\
5.10 \%\end{array}$ \\
\hline & Otras redes & 24 & $5.1 \%$ \\
\hline
\end{tabular}

La mayor parte de las mujeres que integran la muestra son de nacionalidad española (96.9\%), tienen estudios universitarios
(39.7\%) o estudios primarios (34.8\%). El $12.7 \%$ no tiene estudios y sólo el $11.8 \%$ tienen estudios medios. Casi la mitad tiene empleo 
remunerado (48.8\%), estando las 21.80\% desempleadas, aunque también están presentes en la muestra las estudiantes (21.6\%) y las amas de casa (11.8\%). En cuanto a las redes sociales que usan, el $43.7 \%$ utilizan Facebook y Tuenti y el $41.6 \%$ solo Facebook.

\section{Instrumentos}

Se aplicó una encuesta sobre los usos que hacen las mujeres de las redes sociales que incluyó las siguientes secciones:

- Características sociodemográficas y de estudios: se preguntaba a las participantes por su edad, nivel educativo, situación familiar y actividad laboral actual

- Intensidad de uso, que consta de 4 ítems que miden la frecuencia de uso de las redes Facebook, Tuenti, Twitter y LinkedIn con un rango de respuesta que oscila entre 0 (nunca) y 3 (a diario). Para la medida de la variable de intensidad de uso nos apoyamos en estudios previos (Ellison et al., 2007; Steinfield et al., 2008; Valenzuela et al., 2009) en los que la frecuencia de uso se toma como principal indicador

- Motivos de uso, que incluye 14 ítems de respuesta nominal en el que las participantes podían señalar todas las opciones con las que se identificasen. Para asegurar la validez de contenido, se elaboró una lista de control de los motivos de uso aparecidos en investigaciones previas (Arteaga et al., 2014; Hew \& Cheung, 2012; Kwon, D’Angelo \& McLeod, 2013; Yang \& Brown, 2013), completando la lista con otros extraídos de investigaciones centradas en usos de las TIC en personas adultas (Lin, Tang \& Kuo, 2012; Braun, 2013).

- Habilidad de uso, escala elaborada ad hoc a partir de estudios previos (Hargittai, 2010; Livignstone \& Helsper, 2010) está compuesta por 8 ítems de respuesta tipo likert de 5 puntos (desde 0 -nada- hasta 4 -totalmente). Esta escala muestra un coeficiente de fiabilidad de la medida alfa de Cronbach de .863 así como índices óptimos de validez de constructo al presentar una saturación media de los ítems en el componente principal de 0,644 y una desviación típica de .089

- Autonomía de uso, escala elaborada ad hoc a partir de investigaciones previas (Correa, 2010; Hargittai \& Walejko, 2008), está compuesta por 4 ítems de respuesta tipo Likert de 5 puntos (desde 0 -nada- hasta 4 -totalmente-). Aplicado un análisis factorial de componentes principales, la escala muestra una validez de constructo óptima, al presentar todos los ítems índices altos de saturación en el componente principal con una media de .691 y una desviación típica de .040, lo que indica unidimensionalidad. También obtiene un coeficiente aceptable de fiabilidad con un alfa de Cronbach de .776 .

\section{Procedimiento}

Para el trabajo de campo contamos con la colaboración de las Agentes de Igualdad de los Puntos de Información a la Mujer (PIM) de los municipios, a las que se formó en la administración de la encuesta [1]. La estrategia adoptada para aplicar el cuestionario ha sido con presencia de la encuestadora y mediante interacción verbal con el objeto de garantizar la adecuada comprensión de los ítems y su correcta cumplimentación por parte de las encuestadas con independencia de su nivel de estudios. La encuesta se realiza en las dependencias municipales dedicadas a la formación en tecnologías digitales con una duración aproximada de 30 minutos. La recogida de datos se efectuó durante los meses de marzo, abril y mayo de 2012. Las mujeres participantes fueron debidamente informadas del carácter voluntario y anónimo de su participación en el estudio y de los objetivos del mismo, aceptando participar voluntariamente sin recibir compensación a cambio.

Los datos obtenidos han sido tratados con el paquete estadístico SPSS (versión 18 para Windows). A partir de las 14 variables 
nominales sobre motivos de uso incluidas en la encuesta, hemos creado cuatro variables nominales sobre motivos de uso de las redes sociales virtuales (relacional, identidad, entretenimiento e información) producto de su agrupamiento conceptual, con el objeto de operar con ella en los análisis correlacionales multivariantes. Para la agrupación conceptual de las 14 variables de motivos se tienen en cuenta diferentes aportaciones (Arteaga et al., 2014; Dogruer et al., 2011; Hew \& Cheung, 2012; Joinson, 2008; Yang \& Brown, 2013) procediendo a agrupar como motivos de tipo relacional las variables mantener amigos, relacionarme rápido y fluido, compartir experiencias, retomar contactos, hacer amigos, por otro lado, se agrupan en motivaciones relacionadas con la identidad las variables, unirme a grupos, sentirme incluida, darme a conocer $\mathrm{y}$, por último, en el grupo de motivaciones orientadas al entretenimiento y a la obtención de la información se incorporan variables como saber de mi profesión, saber de otras personas, saber sobre mi entorno, ver fotos y videos, entretenimiento, está de moda.

En cuanto a las variables habilidad y autonomía, una vez comprobada la unidimensionalidad de las escalas mediante análisis factorial, se crea una variable de cada una de ellas con la puntuación global a partir del sumatorio de todos los ítems, quedando la variable global de habilidad en una escala ordinal de 0 a 32 puntos y la variable global de autonomía en una escala ordinal de 0 a 16 puntos respectivamente.

En relación al tratamiento estadístico de los datos, hemos aplicado un análisis exploratorio de las variables en estudio para conocer las características de la distribución. Posteriormente, se realiza un análisis bivariante a través de tablas de contingencia para observar la relación entre variables y la prueba de chi-cuadrado para estudiar la incidencia de las variables edad y nivel educativo. Por último, también se realiza un análisis de conglomerados de $\mathrm{K}$ medias (QCluster) para establecer perfiles y un análisis discriminante para caracterizar los perfiles en función de habilidad, autonomía e intensidad de uso.

\section{Análisis y resultados}

Respecto al primer objetivo de nuestra investigación, es decir, conocer los motivaciones de las mujeres de entorno rural para usar las redes sociales virtuales, los datos obtenidos indican que el $75 \%$ de las encuestadas señalan de 5 a 10 motivos diferentes para usarlas, mientras que solo el $14.6 \%$ señalan menos de 5 motivos y el $10.4 \%$ señalan más de 10 motivos distintos. Estos datos sugieren que las mujeres se acercan a las redes sociales virtuales con intereses y motivos variados, posibilitándoles realizar una variedad de actividades e inquietudes.

Los resultados muestran que los motivos de uso más frecuentes son mantener contacto con amistades y familiares, comunicarse rápido y fluido, saber acerca de lo que ocurre en su entorno social, compartir experiencias e información, ver fotos y vídeos de interés, unirse a grupos con intereses comunes y entretenerse. El Gráfico 1 muestra los datos de distribución en la variable motivos de uso. 
Gráfico 1: Porcentaje de mujeres que señalan distintos motivos de uso

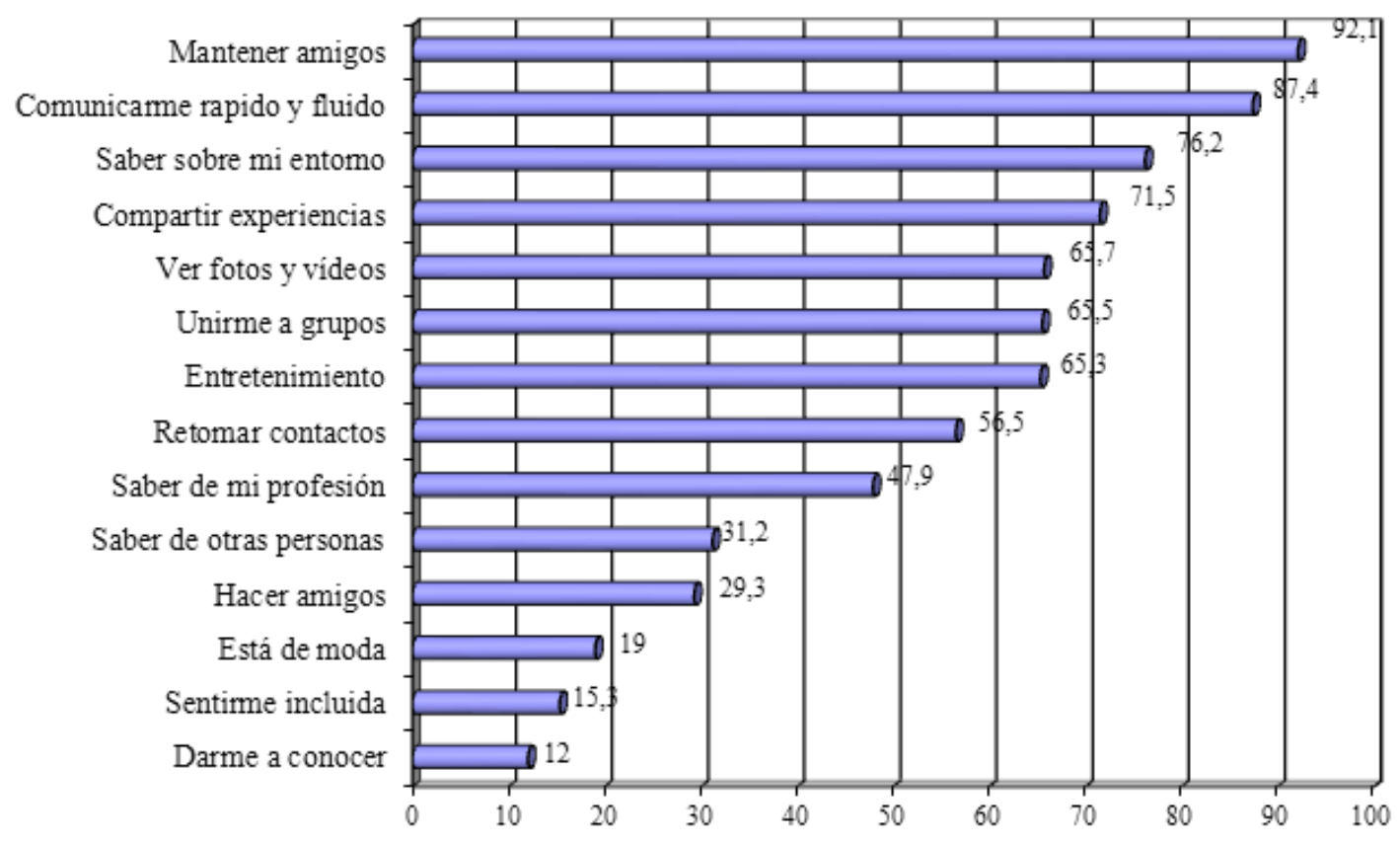

Los resultados también nos muestran que, los motivos de uso que expresan las mujeres encuestadas no difieren en función de la edad y el nivel educativo en términos generales. No obstante, encontramos diferencias en el perfil de mujeres que usan las redes con el propósito de unirse a grupos con intereses comunes y conocer acerca de otras personas en función de la edad, siendo las menores de 40 años las que más la usan con este interés. Asimismo, comunicarse de forma rápida y fluida y

actualizarse profesionalmente son motivaciones propias de mujeres menores de 55 años, siendo una motivación menos frecuente en el grupo de mayor edad. En contraste, los resultados muestran que es más frecuente entre las mujeres de más de 55 años que usen las redes sociales para sentirse socialmente incluidas. La Tabla 2 muestra los resultados del coeficiente de contingencia y chi cuadrado para estas variables.

TABLA 2. Tabla de contingencia de motivos de uso en función de la edad

\begin{tabular}{|c|c|c|c|c|c|c|}
\hline & & $\begin{array}{l}\leq 25 \text { años } \\
(n=117)\end{array}$ & $\begin{array}{c}26 \text { a } 39 \\
(n=153)\end{array}$ & $\begin{array}{c}40 \text { a } 54 \\
(n=152)\end{array}$ & $\begin{array}{c}\geq 55 \text { años } \\
(n=51)\end{array}$ & Significación \\
\hline \multirow[b]{2}{*}{$\begin{array}{l}\text { 12. Unirme a grupos } \\
\text { con intereses afines }\end{array}$} & $\mathrm{Si}$ & 106 (90.6\%) & 103 (67.3\%) & 78 (51.3\%) & $23(45.1 \%)$ & \multirow{2}{*}{$\begin{array}{c}\mathrm{X}(2)=55.796 \\
\mathrm{p}=.000 \\
\text { C. C. }=.325\end{array}$} \\
\hline & No & $11(9.4 \%)$ & $50(32.7 \%)$ & 74 (48.7\%) & 28 (54.9\%) & \\
\hline \multirow{2}{*}{$\begin{array}{l}\text { 10. Comunicarme } \\
\text { rápido y fluido }\end{array}$} & $\mathrm{S}$ & $114(97.4 \%)$ & $139(90.8 \%)$ & $123(80.9 \%)$ & $38(74.5 \%)$ & \multirow{2}{*}{$\begin{array}{c}\mathrm{X}(2)=26.060 \\
\mathrm{p}=.000 \\
\text { C. C. }=, 229\end{array}$} \\
\hline & No & $3(2.6 \%)$ & $14(9.2 \%)$ & 29 (19.1\%) & 13 (25.5\%) & \\
\hline \multirow{2}{*}{$\begin{array}{l}\text { 5. Saber de la vida } \\
\text { de otras personas }\end{array}$} & Si & 57 (48.7\%\%) & $44(28.8 \%)$ & $30(19.7 \%)$ & $16(31.4 \%)$ & \multirow{2}{*}{$\begin{array}{c}\mathrm{X}(2)=26.511 \\
\mathrm{p}=.000 \\
\text { C.C. }=.230\end{array}$} \\
\hline & No & 60 (51.3\%) & 109 (71.2\%) & 122 (80.3\%) & 35 (68.6\%) & \\
\hline \multirow{2}{*}{$\begin{array}{l}\text { 9. Sentirme } \\
\text { socialmente } \\
\text { incluida }\end{array}$} & $\mathrm{Si}$ & $16(13.7 \%)$ & $15(9.8 \%)$ & 23 (15.1\%) & $19(37.3 \%)$ & \multirow{2}{*}{$\begin{array}{c}\mathrm{X}(2)=22.610 \\
\mathrm{p}=.000 \\
\text { C.C. }=.214\end{array}$} \\
\hline & No & $101(86.3 \%)$ & 138 (90.2\%) & 129 (84.9\%) & $32(62.7 \%)$ & \\
\hline \multirow{2}{*}{$\begin{array}{l}\text { 11. Estar actualizada } \\
\text { profesionalmente }\end{array}$} & Si & $65(55.6 \%)$ & $82(53.66 \%)$ & $68(44.7 \%)$ & $11(21.6 \%)$ & \multirow{2}{*}{$\begin{array}{c}\mathrm{X}(2)=19.516 \\
\mathrm{p}=.000 \\
\text { C. C. }=.199\end{array}$} \\
\hline & No & 51 (44.4\%) & 71 (46.4\%) & 84 (55.3\%) & 40 (78.4\%) & \\
\hline
\end{tabular}

También encontramos diferencias en algunas motivaciones de uso en función del nivel de estudios. Concretamente, hacer nuevas amistades y sentirse socialmente incluida son motivaciones menos frecuentes en el grupo de mujeres con estudios universitarios 
que en el resto. La Tabla 3 muestra los para estas variables. coeficientes de contingencia y chi cuadrado

TABLA 3: Tabla de contingencia de motivos de uso según nivel educativo

\begin{tabular}{lcccccc}
\hline & & $\begin{array}{c}\text { Sin estudios } \\
(\mathbf{n}=\mathbf{6 0})\end{array}$ & $\begin{array}{c}\text { Primaria } \\
\mathbf{( n = 1 7 0 )}\end{array}$ & $\begin{array}{c}\text { Secundaria } \\
\mathbf{( n = 5 6 )}\end{array}$ & $\begin{array}{c}\text { Universidad } \\
(\mathbf{n}=\mathbf{1 8 8})\end{array}$ & Significación \\
\hline $\begin{array}{l}\text { 2. Hacer } \\
\text { nuevas } \\
\text { amistades }\end{array}$ & Si & $29(48.3 \%)$ & $57(33.5 \%)$ & $19(33.9 \%)$ & $34(18.1 \%)$ & $\begin{array}{c}\mathrm{X}(2)=23.943 \\
\mathrm{p}=.000\end{array}$ \\
\hline $\begin{array}{l}\text { 9. Sentirme } \\
\text { socialmente } \\
\text { incluida }\end{array}$ & Si & $17(28.3 \%)$ & $30(17.6 \%)$ & $10(17.9 \%)$ & $16(8.5 \%)$ & X. C. $=.219)=15.470$ \\
\hline
\end{tabular}

En resumen, los resultados muestran que un porcentaje muy elevado de las mujeres encuestadas usan las redes sociales no sólo con el interés de mantener relaciones y comunicarse $(97.5 \%)$, sino también para estar informada (90\%) y para divertirse $y$ entretenerse (86\%), siendo menos frecuente usarlas con el propósito de desarrollar y mostrar su identidad digital (76.4\%).

Para identificar perfiles de mujeres a partir de las motivaciones de uso expresadas, realizamos un análisis cluster, encontrando dos grupos bien diferenciados. Mientras las mujeres que se incluyen en el grupo 1 expresan una gran heterogeneidad de motivos de uso, las del grupo 2 usan las redes con una motivación puramente relacional. La prueba de Anova muestra que las diferencias encontradas son significativas en las variables implicadas. La Tabla 4 recoge los resultados de la partición en dos conglomerados de la muestra.

TABLA 4: Resultados de la partición en dos clusters y ANOVA exploratorio

\begin{tabular}{|c|c|c|c|c|c|c|c|c|}
\hline & \multicolumn{2}{|c|}{$\begin{array}{c}\text { Centros de } \\
\text { conglomerados finales }\end{array}$} & \multicolumn{6}{|c|}{ ANOVA } \\
\hline & \multirow[b]{2}{*}{1} & \multirow[b]{2}{*}{2} & \multicolumn{2}{|c|}{ Conglomerado } & \multicolumn{2}{|c|}{ Error } & \multirow[b]{2}{*}{$\mathbf{F}$} & \multirow[b]{2}{*}{ Sig. } \\
\hline & & & $\begin{array}{c}\text { Media } \\
\text { cuadrática }\end{array}$ & Gl & $\begin{array}{c}\text { Media } \\
\text { cuadrática }\end{array}$ & GI & & \\
\hline $\begin{array}{l}\text { Motivo Relacional } \\
\text { (mantener relaciones } \\
\text { y comunicación) }\end{array}$ & 1 & 1 & .822 & 1 & .023 & 476 & 35.958 & .000 \\
\hline Motivo Identidad & 1 & 0 & 31.018 & 1 & .116 & 476 & 267.145 & .000 \\
\hline $\begin{array}{l}\text { Motivo } \\
\text { Entretenimiento }\end{array}$ & 1 & 0 & 19.467 & 1 & .080 & 476 & 242.949 & .000 \\
\hline $\begin{array}{l}\text { Motivo Información } \\
\text { (estar al día) }\end{array}$ & 1 & 0 & 15.241 & 1 & .059 & 476 & 259.661 & .000 \\
\hline
\end{tabular}

Los resultados de un análisis exploratorio bivariante sobre la habilidad y autonomía de uso en cada uno de los grupos de mujeres identificados en función de la motivación, nos muestra que aquellas que hacen un uso más heterogéneo presentan mayores niveles de habilidad y autonomía de uso que aquellas que lo hacen por motivos exclusivamente relacionales. Los Gráficos 2 y 3 muestran el análisis bivariante entre los dos grupos de mujeres. 


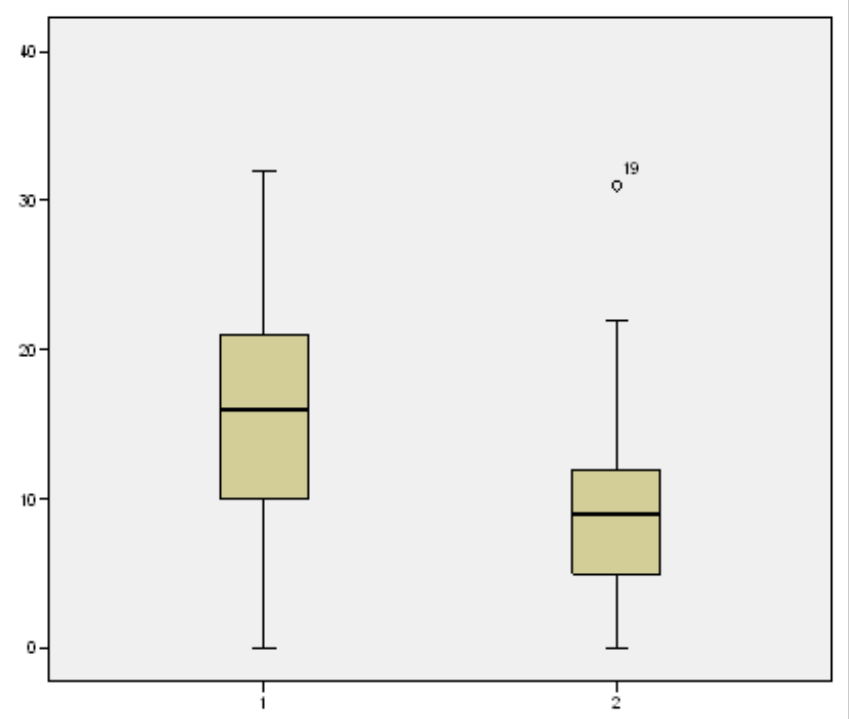

Gráfico 2: Nivel de habilidad según perfiles en motivaciones de uso

Con el objeto de conocer la incidencia de estas variables en la caracterización y exploración de estos dos grupos, realizamos un análisis discriminante, tomando como variable criterio los clusters formados a partir de motivos de uso y como variables predictoras la habilidad, autonomía e intensidad de uso. La ecuación discriminante, aunque muestra una baja correlación canónica de .275, presenta una adecuada bondad de ajuste (Landa de Wilk $=.924 ; \mathrm{p}=.000$ ), expresando que la variable habilidad en el uso de las redes sociales es la más determinante. La ecuación discriminante resultante se simplifica en la siguiente fórmula: $\mathrm{y}=.142$ Habilidad -2.093 . La ecuación excluye otras variables predictoras por la multicolinealidad entre las

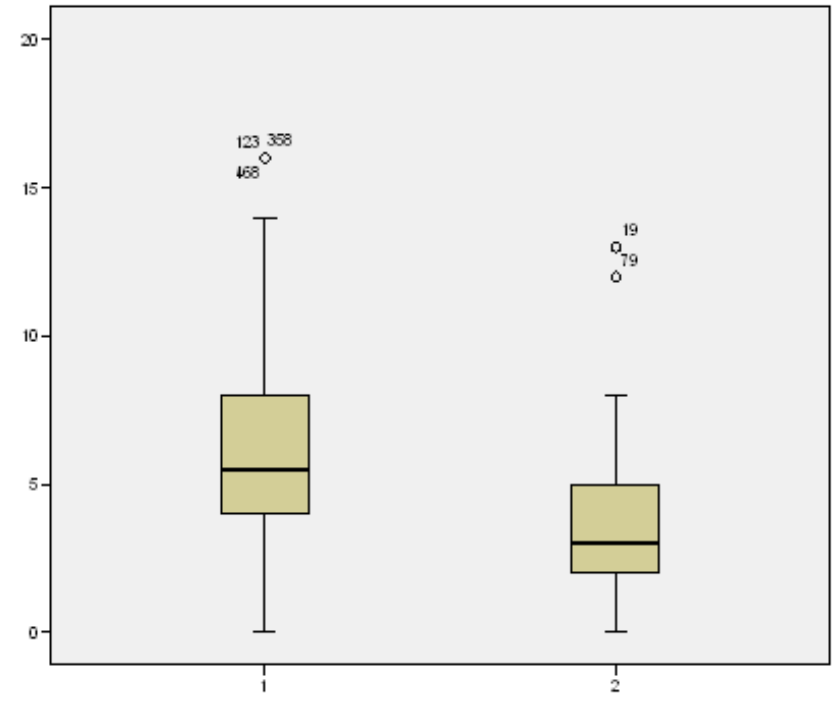

Gráfico 3: Nivel de autonomía según perfiles en motivaciones de uso

mismas asumiendo la habilidad la función representativa del conjunto, por lo que el análisis de la matriz de estructura total puede aportar información clave para conocer el papel de las demás variables predictoras.

La matriz de estructura total muestra el peso de cada variable en la diferenciación de perfiles de mujeres en el uso de las redes sociales, encontrando que la habilidad de uso es la que más protagonismo asume en la diferenciación de perfiles de mujeres en cuanto a motivos de uso, seguido de autonomía y siendo la intensidad de uso la variable que menos explica la diferenciación en perfiles de mujeres según motivos de uso. La Tabla 5 muestra la matriz de estructura total de la función discriminante.

TABLA 5. Matriz de estructura total de la función discriminante

\begin{tabular}{lc}
\hline Matriz de estructura totall & Función \\
\hline Habilidad en el uso de redes & $\mathbf{1}$ \\
Autonomía en el uso de redes(a) & 1.000 \\
Intensidad de uso Facebook(a) & .549 \\
\hline
\end{tabular}

Aplicada la prueba $t$ de Student para la comparación de medias se observa que existen diferencias estadísticamente significativas entre los perfiles de mujeres en lo que se refiere a habilidad y autonomía de uso ( $\mathrm{t}_{\text {habilidad }}=6.940 ; \mathrm{p}=.000 ; \mathrm{t}_{\text {autonomía }}=$ 4.863; $\mathrm{p}=.000$ ) pero no se observan en cuanto a intensidad ( $\mathrm{t}_{\text {intensidad }}=1.764 ; \mathrm{p}=$ $.082)$. 
No se observan diferencias significativas en los perfiles de mujeres en función del nivel educativo (chi cuadrado $=1.082 ; \mathrm{p}=.782$ ), aunque sí en función de la edad (chi cuadrado $=13.029 ; \mathrm{p}=.005$ ), encontrando que hay proporcionalmente más mujeres de 40 a 54 años en el grupo que usa las redes con motivos exclusivamente relacionales.

\section{Discusión y conclusiones}

El propósito general de nuestra
investigación ha sido analizar las
motivaciones de uso de las mujeres de
entorno rural y caracterizarlas en diferentes
perfiles con el objeto de explorar la relación
entre motivos de uso y habilidad, autonomía e
intensidad de uso. El fin último, es
documentar patrones en las formas de usar las
redes para utilizarlos en el diseño de
propuestas educativas.

Respecto al primer objetivo del estudio, los resultados indican que las mujeres en general muestran una gran variedad de motivos en el uso de las redes sociales, siendo los motivos relacionales, de información y de entretenimiento los más frecuentes. Esto coincide con estudios previos (Arteaga et al., 2014; Dogruer et al., 2011; Hew \& Cheung, 2012; Yang \& Brown, 2013) que habían identificado estos tres tipos de motivos. Sin embargo, estos resultados concuerdan solo parcialmente con otras investigaciones (Lin \& Lu, 2011; Dias, 2012; Rebollo \& Vico, 2014) en las que se habían encontrado que los motivos de uso más frecuentes eran relacionarse socialmente y entretenerse. En nuestro estudio, encontramos que los motivos de uso vinculados a una función informativa también asumen un papel importante en el uso que hacen las mujeres de entorno rural de las redes sociales aprovechando su capital social. En nuestro estudio hemos observado que las mujeres que usan las redes con el propósito de unirse a grupos con intereses comunes son menores de 40 años. De acuerdo con Joinson (2008) el uso de las redes está relacionado con el refuerzo de la identidad en actividades que entrañan participación en grupos con intereses afines. Por tanto sabemos que el uso de las redes está vinculado a la formación y refuerzo identitario, lo que supone un elemento muy importante a tener en cuenta en los diseños tecnológicos de las redes y en las propuestas educativas que las incorporen.

En cuanto al segundo objetivo de nuestra investigación encaminado a determinar si existían patrones diferenciales en las motivaciones de uso de las redes entre las mujeres rurales $\mathrm{y}$, en caso afirmativo, caracterizarlos a partir de la intensidad, habilidad y autonomía de uso, hemos encontrado dos perfiles de mujeres significativamente diferentes; mujeres que usan las redes sociales con una gran diversidad de motivaciones y que muestran un mayor nivel de habilidad, autonomía e intensidad en el uso de las redes y, mujeres que usan las redes exclusivamente por motivos relacionales y que expresan un menor nivel en habilidad, autonomía e intensidad de uso de las redes sociales. Estos resultados concuerdan con los obtenidos en investigaciones previas (Hargittai, 2010; Livingstone \& Helsper, 2007; Smok et al. 2008) que señalan la relevancia que tiene el uso diversificado de Internet para la inclusión digital.

Nuestro estudio también muestra el importante papel que asume la habilidad de uso en la diferenciación de perfiles. Las investigaciones anteriores sobre habilidad y autonomía de uso (Hargittai \& Walejko, 2008; Hargittai, 2010; Correa, 2010) también han constatado el papel mediador que tiene la habilidad en el desarrollo de una diversidad de actividades virtuales, constatando incluso este papel mediador para explicar las actividades creativas de las mujeres en las redes. Esto supone interesantes claves para el diseño de propuestas educativas y redes de aprendizaje que incorporen por ejemplo estrategias como los círculos digitales de apoyo (Godfrey \& Johnson, 2009) al poder contar con mujeres que muestran una mayor habilidad de uso y tienen experiencia en actividades creativas en entornos en red.

Estos resultados indican que la experiencia e intensidad en el uso de las redes sociales 
está asociada a la aparición de nuevas motivaciones, es decir, que la motivación inicial de mantener contacto con amigos y familiares se diversifica en una variedad de formas de uso que aumenta con la experiencia de uso, ofreciéndoles una rica gama de actividades y prácticas. Los hallazgos de otros estudios previos también apuntaban en esta misma línea (Livingstone \& Helsper, 2007; Steinfield et al., 2008). Esto tiene interesantes aplicaciones para el diseño de las redes de aprendizaje en relación con los planteamientos de Sloap y Berlanga (2011). Estos autores consideran que las redes de aprendizaje deben obedecer a la creación conjunta y ser fruto de un proceso interactivo y participativo, priorizando enfoques centrados en el usuario a la hora de crear redes de aprendizaje concretas. Así, sería clave el diseño de redes de aprendizaje donde se parta de las motivaciones de uso concretas, de los perfiles de las participantes y de las tecnologías de que disponen en su ámbito local.

Los resultados muestran diferencias significativas en el perfil de mujeres en función de la edad, encontrando que hay más mujeres de 40 a 54 años en el grupo que usa las redes con motivos exclusivamente relacionales que muestran menor nivel de habilidad, autonomía e intensidad de uso. Otros estudios previos señalan a las personas mayores como un grupo vulnerable a la exclusión digital (Brandtzæga, Heim \& Karahasanovic, 2011; Braun, 2013; Dias, 2012). En nuestro estudio, encontramos que las mujeres de comunidades rurales de 40 a 54 también forman parte de este grupo. La brecha generacional ha sido documentada con anterioridad en relación con la inclusión digital (DiMaggio, Hargittai, Celeste \& Shafer, 2004; Hargittai 2002; van Dijk, 2005), aunque no se ha abordado específicamente en relación con el uso de las redes sociales donde la mayor parte de los estudios se han centrado en estudiantes con edades similares (Arteaga et al., 2014; Dogruer et al., 2011; Smok et al., 2011; Steinfield et al., 2008; Ellison et al., 2007). Estos resultados sobre el uso de las redes sociales virtuales por parte de mujeres de entornos rurales de nuestro estudio apuntan en la misma línea de estas investigaciones. Los resultados no muestran diferencias en los perfiles en función del nivel educativo.

En suma, los resultados sugieren la necesidad de promover una variedad de usos de las redes sociales por parte de las mujeres en comunidades rurales y plantear propuestas educativas orientadas a diferentes perfiles que partan de sus motivaciones de uso. Diseñar estrategias educativas sensibles a estos perfiles supondrá una forma de aumentar no solo su capital social sino también su alfabetización e inclusión digital. Favorecer una participación más activa y diversa por parte de las mujeres de zonas rurales en las redes pasa por el planteamiento de propuestas pedagógicas especialmente encaminadas a constituir círculos de apoyo en los que en contacto con otras mujeres que disponen de mayores niveles habilidad y autonomía de uso. Los hallazgos permiten repensar desde un punto de vista educativo el establecimiento de puentes entre el aprendizaje formal y el informal para la mejora de la alfabetización digital de las mujeres de zonas rurales.

\section{Referencias}

Area, M. \& Pessoa, T. (2012). De lo sólido a lo líquido: Las nuevas alfabetizaciones ante los cambios culturales de la Web 2.0. Comunicar, XIX, 38, 13-20. DOI:10.3916/C38-2011-02-01

Arteaga, R., Cortijo, V. \& Javed, U. (2014). Students' perceptions of Facebook for academic purposes. Computers \& Education, 70, 138-149. DOI:10.1016/j.compedu.2013.08.012

Attwell, G. (2007). Personal Learning Environments. The future of e-learning? eLearning Papers, 2(1) Recuperado de http://www.elearningeuropa.info/files/media/me dia11561.pdf

Brandtzæga P.B., Heim, J. \& Karahasanovic, A. (2011). Understanding the new digital divide. A typology of Internet users in Europe. International Journal of Human Computer Studies, $\quad 69(3)$, 123-138. DOI:10.1016/j.ijhcs.2010.11.004

Braun, M. T. (2013). Obstacles to social networking website use among older adults. 
Computers in Human Behavior, 29, 673-680. DOI:10.1016/j.chb.2012.12.004

Carpenter, J. M., Green, M. C. \& LaFlam, J. (2011). People or profiles: Individual differences in online social networking use. Personality and Individual Differences, 50(5), 538541.DOI:10.1016/j.paid.2010.11.006

Castaño, C. (2009). La segunda brecha digital y las mujeres jóvenes. Quaderns de la Mediterrània, 11, 218-224. Recuperado dehttp://www.iemed.org/publicacions/quaderns/ 11/41 la segunda brecha digital.pdf

Chen, L.Y, Hsiao, B., Chern, C.C. \& Chen, H.G. (2014). Affective mechanisms linking Internet use to learning performance in high school students: A moderated mediation study. Computers in Human Behavior, 35, 431-443. DOI: 10.1016/j.chb.2014.03.025

Cheung, C. M. K., Chiu, P.Y. \& Lee, M. K. O. (2011). Online social networks: Why do students use facebook? Computers in Human Behavior, 27(4), 13371343.DOI:10.1016/j.chb.2010.07.028

Clipson, T., Wilson, S. A. \& DuFrene, D. D. (2010). The social networking arena: battle of the sexes. Business Communication Quarterly, 75 (1), 1-5.

Coiro, J., Knobel, M., Lankshear, C., \& Leu, D. J. (Eds). (2008). Handbook of research in new literacies. Mahwah, NJ: Lawrence Erlbaum Associates.

Correa, T. (2010). The Participation Divide Among “Online Experts”: Experience, Skills and Psychological Factors as Predictors of College Students' Web Content Creation. Journal of Computer-Mediated Communication, 16, 71-92. DOI:10.1111/j.10836101.2010.01532.x

Dabbagh, N., \& Kitsantas, A. (2012). Personal Learning Environments, social media, and selfregulated learning: A natural formula for connecting formal and informal learning. The Internet and Higher Education, 15(1), 3-8. DOI:10.1016/j.iheduc.2011.06.002

Dias, I. (2012). O uso das tecnologías digitais entre os seniores. Motivações e intereses. Sociologia, Problemas e Práticas, 68, 51-77. DOI: $10.7458 /$ SPP201268693

DiMaggio, P., Hargittai, E., Celeste, C., \& Shafer, S. (2004). Digital Inequality: From Unequal Access to Differentiated Use. In K. Neckerman
(Ed.), Social Inequality (355-400). New York: Russell Sage Foundation.

Dogruer, N., Menevis, I. \& Eyyam, R. (2011). What is the motivation for using Facebook? Procedia Social and Behavioral Sciences, 15, 2642-2646. DOI:10.1016/j.sbspro.2011.04.162

Ellison, N. B., Steinfield, C. \& Lampe, C. (2007). The benefits of Facebook "friends:" Social capital and college students' use of online social network sites. Journal of Computer-Mediated Communication, 12(4), 1143-1168.

Falk, I. \& Kilpatrick, S. (2000). What is social capital? A study of interaction in a rural community. Sociologia Ruralis, 40 (1), 87-110.

Freese, J., Rivas, S. \& Hargittai, E. (2006). Cognitive ability and Internet use among older adults. Poetics, 34, 236-249. DOI:10.1016/j.poetic.2006.05.008

García, A., López-de-Ayala, M.C. \& Catalina, B. (2013). Hábitos de uso en Internet y en las redes sociales de los adolescentes españoles. Comunicar, XXI (41), 195-204.

Gómez, M., Roses, S. \& Farias, P. (2012). El uso académico de las redes sociales en universitarios. Comunicar, XIX(38), 131-138. DOI: 10.3916/C38-2011-03-04

Godfrey, M. \& Johnson, O. (2009). Digital circles of support: Meeting the information needs of older people. Computers in Human Behavior, 25, 633-642. DOI:10.1016/j.chb.2008.08.016

Greenhow, C. \& Burton, L. (2011). Help from my "Friends:" Social capital in the social network sites of low-income high school students. Journal of Educational Computing Research, 45(2), 223-245.

Greenhow, C. \& Robelia, E. (2009). Informal learning and identity formation in online social networks. Learning, Media and Technology, 34(2), 119-140.

Hargittai, E. \& Hinnant, A. (2008). Digital Inequality: Differences in Young Adults' Use of the Internet. Communication Research, 35(5), 602-621. DOI:10.1177/0093650208321782

Hargittai, E. \& Hsieh, Y.P. (2010).Predictors and consequences of differentiated practices on social network sites.Information, Communication \& Society, 13(4), 515-536. DOI: 10.1080/13691181003639866

Hargittai, E. \& Walejko, G. (2008). The participation divide: Content creation and sharing in the digital age. Information, 
Communication \& Society, 11 (2), 239-256. DOI: 10.1080/13691180801946150

Hargittai, E. (2002). Second-level digital divide: differences in people's online skills. First Monday, 7 (4), 1-15

Hargittai, E. (2008). Whose Space? Differences Among Users and Non-Users of Social Network Sites. Journal of Computer-Mediated Communication, $\quad 13, \quad 276-297$. DOI:10.1111/j.1083-6101.2007.00396.x

Hargittai, E. (2010). Digital Natives? Variation in Internet Skills and Uses among Members of the “Net Generation'”. Sociological Inquiry, 80 (1), 92-113. $\quad$ DOI: $\quad 10.1111 / \mathrm{j} .1475-$ 682X.2009.00317.x

Hew, K.F. \& Cheung, W.S. (2012). Students' use of asynchronous voice discussion in a blendedlearning environment: A study of two undergraduate classes. The Electronic Journal of e-Learning, 10(4) 360-367.

Hsieh, Y.P. (2012). Online social networking skills: The social affordances approach to digital inequality. First Monday, 17 (4), 1-12.

Imhof, M., Vollmeyer, R. \& Beierlein, C. (2007). Computer use and the gender gap: The issue of access, use, motivation, and performance. Computers in Human Behavior, 23(6), 28232837.DOI: 10.1016/j.chb.2006.05.007

Jackson, L. A., Ervin, K. S., Gardner, P. D., \& Schmitt, N. (2001). Gender and the Internet: Women communicating and men searching. Sex Roles, 44(5/6), 363-379. $\quad$ DOI: 10.1023/A:1010937901821

Joinson, N. A. (2008). Looking at', 'Looking up' or 'Keeping up with' People? Motives and uses of Facebook. CHI 2008 Proceedings: 10271036.

Kwon, M.W. D’Angelo \& McLeod, J. D.M. (2013). Facebook use and social capital: To bond, to bridge, or to escape. Bulletin of Science, Technology \& Society, 33(5-6).

LaRose, R., Greggb, J.L., Stroverc, S., Straubhaarc, J. \& Carpenterd, S. (2007) Closing the rural broadband gap: Promoting adoption of the Internet in rural America. Telecommunications Policy,31, 359-373. DOI: 10.1016/j.telpol.-2007.04.004

Ley 45/2007, de 13 de diciembre, para el desarrollo sostenible del medio rural. Boletín Oficial del Estado, 299, de 15 de diciembre de 2007.
Lin, C., Tang, W. \& Kuo, F. (2012). Mommy Wants to Learn the Computer: How MiddleAged and Elderly Women in Taiwan Learn ICT Through Social Support. Adult Education Quarterly, 62 (1), 73-90. DOI: 10.1177/0741713610392760

Lin, K.Y. \& Lu, H.P. (2011). Why people use social networking sites: An empirical study integrating network externalities and motivation theory. Computers in Human Behavior, 27(3), 1152-1161.DOI: 10.1016/j.chb.2010.12.009

Livingstone, S. \& Helsper, E. (2007). Gradations in digital inclusion: children, young people and the digital divide. New Media \& Society, 9(4), 671- 696. DOI: $10.1177 / 1461444807080335$

Livingstone, S. \& Helsper, E. (2010).Balancing opportunities and risks in teenagers' use of the internet: the role of online skills and internet self-efficacy. .New media \& society, 12 (2), 309329. DOI: $10.1177 / 1461444809342697$

Livingstone, S., Van Couvering, E. \& Thumim, N. (2008). Converging traditions of research on media and information literacies: disciplinary, critical, and methodological issues. En J. Coiro, M., Knobel, C. Lankshear \& D. J. Leu, (Eds.) Handbook of research on new literacies. Mahwah, NJ: Lawrence Erlbaum Associates.

Martinez-Pecino, R., Delerue, A. \& Silva, P. (2013.). Portuguese older people and the Internet: Interaction, uses, motivations, and obstacles. Communications - The European Journal of Communication Research, 38(4), 331346. DOI: 10.1515/commun-2013-0020

Mazman S.G. \& Usluel, Y. K. (2011). Gender differences in using social networks. TOJET: The Turkish Online Journal of Educational Technology, 10 (2), 133-139. Recuperado dehttp://goo.gl/8042eT

Meyers, E.M., Erickson, I. \& Small, R.V. (2013). Digital literacy and informal learning environments: an introduction, Learning, Media and Technology, 38(4), 355-367, DOI: 10.1080/17439884.2013.783597

Nadkarni, A. \& Hofmann, S. (2011). Why do people use Facebook? Personality and Individual Differences, 52, 243-249. DOI: 10.1016/j.paid.2011.11.007

Notley, T. (2009). Young People, Online Networks, and Social Inclusion. Journal of Computer-Mediated Communication, 14, 12081227. DOI:10.1111/j.1083-6101.2009.01487.x 
Notley, T.M. \& Foth, M. (2008). Extending Australia's digital divide policy: an examination of the value of social inclusion and social capital policy frameworks. Australian Social Policy, 7, $1-24$.

Novo-Corti, I., Varela-Candamio, L. \& GarcíaÁlvarez, T. (2014). Breaking the walls of social exclusion of women rural by means of ICTs: The case of "digital divides" in Galician. Computers in Human Behavior, 30, 497-507. DOI: 10.1016/j.chb.2013.06.017

Papacharissi, Z. \& Mendelson, A. (2011). Toward a new(er) sociability: uses, gratifications and social capital on Facebook. En S. Papathanassopoulos (Ed.), Media Perspectives for the 21st Century. New York: Routledge (pp. 212-230).

Pfeil, U., Arjan, R. \& Zaphiris, P. (2009). Age differences in online social networking. A study of user profiles and the social capital divide among teenagers and older users in MySpace. Computers in Human Behavior, 25(3), 643-654. DOI: 10.1016/j.chb.2008.08.015

Rebollo-Catalán, A. \& Vico, A. (2014). El apoyo social percibido como factor de la inclusión digital de las mujeres de entorno rural en las redes sociales virtuales. Comunicar, 43, 173180. DOI: $10.3916 / C 43-2014-17$

Rubio, M. J. y Escofet, A. (2013). Estudio sobre los usos de las TIC y el empoderamiento de las mujeres. Revista Iberoamericana de Educación, 62(3), 1-13.

Seidman, G. (2013). Self-presentation and belonging on Facebook: How personality influences social media use and motivations. Personality and Individual Differences, 54, 402407. DOI: 10.1016/j.paid.2012.10.009

Selwyn, N. \& Facer, K. (2007). Beyond the digital divide: Rethinking digital inclusion for the 21st century. Futurelab: Bristol.

Sloep, P. \& Berlanga, A. (2011). Redes de aprendizaje, aprendizaje en red. Comunicar, XIX(37), 55-64. DOI: 10.3916/C37-2011-02-05

Smoke, A.D., Ellison, N.B., Lampe, C. \& Wohn, D.Y. (2011). Facebook as a toolkit: A uses and gratification approach to unbundling feature use. Computers in Human Behavior, 27, 23222329.DOI: 10.1016/j.chb.2011.07.011

Staksrud, E., Ólafsson, K. \& Livingstone, S. (2013). Does the use of social networking sites increase children's risk of harm? Computers in
Human Behavior, 29, 40-50. DOI: 10.1016/j.chb.2012.05.026

Steinfield, C., Ellison, N. B. \& Lampe, C. (2008). Social capital, self-esteem, and use of online social network sites: A longitudinal analysis. Journal of Applied Developmental Psychology, 29(6), 434-445. DOI: 10.1016/j.appdev.2008.07.002

Subrahmanyam, K., Reich, S.M., Waechter, N., Espinoza, G. (2008). Online and offline social networks: Use of social networking sites by emerging adults. Journal of Applied Developmental Psychology, 29, 420-433. DOI: 10.1016/j.appdev.2008.07.003

Tosun, L.P. (2012). Motives for Facebook use and expressing "true self" on the Internet. Computers in Human Behavior, 28, 1510-1517. DOI: 10.1016/j.chb.2012.03.018

Valenzuela, S., Park, N. \& Kee, K. (2009). Is there social capital in a social network site?: Facebook use and college students' life satisfaction, trust, and participation. Journal of Computer-Mediated Communication, 14, 875901. DOI: 10.1111/j.1083-6101.2009.01474.X

vanDeursen, A.J. \& van Dijk, J.A. (2013). The digital divide shifts to differences in usage. New media \& society, 0(0) 1-20. DOI: 10.1177/1461444813487959

vanDijk, J.A. (2006). Digital divide research, achievements and shortcomings. Poetics, 34, 221-235. DOI: 10.1016/j.poetic.2006.05.004

vanDijk, J.A. (2005). The deepening divide: Inequality in the information society. Thousand Oaks: Sage Publications.

Verges, N. (2012). De la exclusión a la autoinclusión de las mujeres en las TIC. Motivaciones, posibilitadores y mecanismos de autoinclusión. Athenea Digital, 12 (3), 129-150.

Vergés, N., Hache, A. \& Cruells, E. (2011). Indagando en la relevancia de internet en el acceso, uso y deseos de las TIC por parte de las mujeres en las TIC. TESI, 12 (2), 105-121.

Yang, C.C \& Brown, B. (2013). Motives for using facebook, patterns of Facebook activities, and late adolescents. Social adjustment to college. Youth Adolescence, 42, 403-416. DOI: 10.1007/s10964-012-9836-X

Zillien, N \& Hargittai, E. (2009). Digital distinction: status-specific Internet uses. Social ScienceQuarterly, 90(2), 274-291 


\section{NOTAS}

${ }^{[1]}$ Esta encuesta es más amplia que lo que se recoge en este artículo, incorporando otras dimensiones para el estudio del uso que hacen las mujeres de las redes sociales, ya que forma parte del proyecto de Investigación de Excelencia, financiado a través de una convocatoria pública, titulado "Calidad Relacional, Inmersión Digital y Bienestar Social desde una Perspectiva de Género. Una Aplicación de las Redes Sociales Online en la Mujer Rural Andaluza”, vigente en la actualidad.

Este trabajo se enmarca en el Proyecto de Investigación de Excelencia titulado Calidad Relacional, Inmersión Digital y Bienestar Social desde una Perspectiva de Género. Una Aplicación de las Redes Sociales Online en la Mujer Rural Andaluza, financiado en la convocatoria de Proyectos de Excelencia 2011/2015 (P10-SEJ-5801).

\begin{tabular}{l|l|}
\hline \multicolumn{1}{c}{ Autor } & To know more / Saber más \\
\hline Jiménez-Cortés, Rocío (rjimenez@us.es). \\
Profesora Ayudante Doctora con acreditación de Profesora Contratada \\
Doctora. Su área de interés se vincula al estudio de las tecnologías desde \\
una perspectiva de género. Forma parte como investigadora principal del \\
proyecto de I+D (2013-2016) titulado “Las mujeres como tejederas de \\
las redes sociales: estrategias relacionales e inclusión digital”. Es la \\
autora de contacto para este artículo. Su dirección postal es Dpto. \\
Métodos de Investigación y Diagnóstico en Educación. Facultad de \\
Ciencias de la Educación Universidad de Sevilla, C/ Pirotecnia, s/n, CP \\
41013, Sevilla (España). \\
\hline Rebollo-Catalán, Ma Ángeles (rebollo@us.es) \\
Profesora Titular de Universidad en la Universidad de Sevilla (España). \\
Su principal línea de investigación se centra en Género, Tecnología y \\
Educación. Su dirección postal es Dpto. Métodos de Investigación y \\
Diagnóstico en Educación. Facultad de Ciencias de la Educación \\
Universidad de Sevilla, C/ Pirotecnia, s/n, CP 41013, Sevilla (España) \\
\hline García-Pérez, Rafael (rafaelgarcia@us.es). \\
Profesor Titular de Universidad en la Universidad de Sevilla (España). \\
Su línea de investigación se centra en Técnicas e Instrumentos de \\
Diagnóstico sobre Género, Tecnología y Educación. Su dirección postal \\
es Dpto. Métodos de Investigación y Diagnóstico en Educación. \\
Facultad de Ciencias de la Educación Universidad de Sevilla, C/ \\
Pirotecnia, s/n, CP 41013, Sevilla (España) \\
\hline Buzón-García, Olga (obuzon@us.es). \\
Profesora asociada de Tecnología Educativa. Su línea de investigación \\
principal es E-learning y TIC en Educación. Su dirección postal es Dpto. \\
Didáctica y Organización Educativa. Facultad de Ciencias de la \\
Educación Universidad de Sevilla, C/ Pirotecnia, s/n, CP 41013, Sevilla \\
(España)
\end{tabular}




\section{RELIEVE}

\section{Revista ELectrónica de Investigación y EValuación Educativa E-Journal of Educational Research, Assessment and Evaluation}

[ISSN: 1134-4032]

(C) Copyright, RELIEVE. Reproduction and distribution of this articles it is authorized if the content is no modified and their origin is indicated (RELIEVE Journal, volume, number and electronic address of the document).

(C) Copyright, RELIEVE. Se autoriza la reproducción y distribución de este artículo siempre que no se modifique el contenido y se indique su origen (RELIEVE, volumen, número y dirección electrónica del documento). 